\title{
Effects of immunization against PCSK9 in an experimental model of breast cancer
}

\author{
Amir Abbas Momtazi-Borojeni ${ }^{1,2}$, Maryam Ebrahimi Nik ${ }^{3}$, Mahmoud Reza Jaafari ${ }^{4,5}$, \\ Maciej Banach ${ }^{6,7}$, Amirhossein Sahebkar,8,9
}

\author{
${ }^{1}$ Nanotechnology Research Center, Bu-Ali Research Institute, Mashhad University \\ of Medical Sciences, Mashhad, Iran \\ ${ }^{2}$ Department of Medical Biotechnology, Student Research Committee, Faculty \\ of Medicine, Mashhad University of Medical Sciences, Mashhad, Iran \\ ${ }^{3}$ Nanotechnology Research Center, Student Research Committee, Pharmaceutical \\ Technology Institute, Mashhad University of Medical Sciences, Mashhad, Iran \\ ${ }^{4}$ Nanotechnology Research Center, Pharmaceutical Technology Institute, Mashhad \\ University of Medical Sciences, Mashhad, Iran \\ ${ }^{5}$ Biotechnology Research Center, Pharmaceutical Technology Institute, Mashhad \\ University of Medical Sciences, Mashhad, Iran \\ ${ }^{6}$ Department of Hypertension, WAM University Hospital, Medical University of Lodz, \\ Lodz, Poland \\ 'Polish Mother's Memorial Hospital Research Institute (PMMHRI), Lodz, Poland \\ ${ }^{8}$ Neurogenic Inflammation Research Center, Mashhad University of Medical Sciences, \\ Mashhad, Iran \\ ${ }^{9}$ School of Pharmacy, Mashhad University of Medical Sciences, Mashhad, Iran
}

Submitted: 25 February 2019

Accepted: 5 April 2019

Arch Med Sci 2019; 15 (3): 570-579

DOI: https://doi.org/10.5114/aoms.2019.84734

Copyright @ 2019 Termedia \& Banach

\begin{abstract}
Introduction: Inhibition of proprotein convertase subtilisin/kexin 9 (PCSK9) is an efficient strategy for lowering low-density lipoprotein cholesterol (LDL-C). There are, however, scant data on the efficacy and safety of PCSK9 inhibitors in non-cardiovascular diseases, particularly cancer. The present study aimed to evaluate the effect of PCSK9 inhibition using a nanoliposomal antiPCSK9 vaccine on cancer behavior and endpoints in mice bearing breast tumor.
\end{abstract}

Material and methods: To induce antiPCSK9 antibody in vivo, a nanoliposomal antiPCSK 9 vaccine absorbed on $0.4 \%$ alum adjuvant was used. To induce tumor, BALB/c mice were subcutaneously inoculated with 4T1 breast carcinoma cells. After the tumor mass was palpable (approximately $10 \mathrm{~mm}^{3}$ ), the mice were randomly divided into four groups and subjected to different treatment protocols: (1) PBS (untreated control), (2) vaccine group, (3) combination of vaccine and Doxil, and (4) Doxil (positive control) group. Vaccine was subcutaneously administered to mice four times at 2-week intervals. Two weeks after the last administration, the vaccinated and non-vaccinated mice were subcutaneously inoculated with 4T1 breast carcinoma cells. To evaluate therapeutic efficacy, mouse body weight, tumor size, and survival were monitored every other day for 60 days.

Results: The nanoliposomal antiPCSK9 vaccine was found to efficiently induce specific antibodies against PCSK9 in BALB/C mice, thereby decreasing plasma levels of PCSK9 and inhibiting its function. Tumor size analysis showed that time to reach endpoint (TTE) of the vaccine, combination, Doxil, and control groups was $47 \pm 10,57 \pm 4,60 \pm 4$ and $39 \pm 9$ days, respectively. Rate of tumor growth in vaccine, combination and Doxil groups was decreased by $21 \%, 48 \%$ and $53 \%$, respectively, compared to the control group. Lifespan was increased by $4.2 \%$ in the vaccine group, compared with the

\author{
Corresponding author: \\ Dr. Amirhossein Sahebkar \\ Biotechnology Research \\ Center \\ Pharmaceutical \\ Technology Institute \\ Mashhad University \\ of Medical Sciences \\ Mashhad 9177948564 \\ Iran \\ E-mail: sahebkara@mums. \\ ac.ir; \\ amir_saheb2000@yahoo.com
}


control group. Additionally, the survival in the combination and Doxil groups was significantly higher than the vaccine and control groups.

Conclusions: Our results revealed that PCSK9 inhibition may moderately improve breast cancer outcomes while having no harmful effects in tumor-bearing mice.

Key words: breast cancer, liposome, nanoparticle, PCSK9, vaccine.

\section{Introduction}

Breast cancer and cardiovascular disease share many metabolic risk factors, such as diet and obesity [1]. Cancer cells are known to have excessive uptake of exogenous cholesterol $[2,3]$ and increased endogenous lipid biosynthesis [4] leading to an abnormal accumulation of cholesterol $[5,6]$. In vitro studies have shown that exposure to low-density lipoprotein (LDL) can induce proliferation and migration of various breast cancer cells derived from different tumor subtypes and stages [7]. In vivo studies modeling high levels of plasma cholesterol have demonstrated larger and more proliferative breast tumors in hypercholesterolemic mice, which also harbor more frequent lung metastases [7]. Suppression of cholesterol storage machinery may decrease cellular proliferation in breast cancer [8]. Nevertheless, observational studies have reported contradictory associations between circulating lipids and breast cancer risk [9-15]. However, a recent Mendelian randomization study has shown a causal and direct association between raised $L D L$-cholesterol ( $L D L-C)$ levels and the risk of breast cancer [16].

LDL-C is principally removed from bloodstream by the liver's LDL receptor (LDLR) that is mainly regulated by proprotein convertase subtilisin/kexin 9 (PCSK9). Hence, PCSK9 has a determinant role in regulating plasma levels of LDL-C $[17,18]$. Early studies have shown that gain-of-function mutations in PCSK9 were causatively associated with increased plasma levels of LDL-C [19], while loss-of-function mutations were associated with hypocholesterolemia and a reduced risk of coronary artery disease [2022]. This association has also been confirmed in proof-of-concept clinical trials [23-30]. Of note, it was recently reported that LDL-raising genetic variants of PCSK9 were associated with a higher risk of breast cancer, while LDL-lowering variants mimicking PCSK9 inhibitors were found to have significant association with a lower risk of breast cancer occurrence [16]. Nonetheless, no evidence is available on the efficacy and safety of PCSK9 inhibitors in non-cardiovascular diseases, particularly cancer.

The famous FDA-approved monoclonal antibodies (mAbs) alirocumab [31, 32] and evolocumab
$[33,34]$ are currently the most effective PCSK9 inhibitors on the market for reducing plasma LDL-C in patients with hypercholesterolemia. AntiPCSK9 vaccines are potential alternatives for PCSK9 mAbs, which can theoretically provide therapeutic effects the same as those attained with mAbs but with advantages such as reduced frequency of injections, and reduced risk of eliciting drug-neutralizing antibodies [35-37]. We previously developed a new generation of antiPCSK9 vaccines that could efficiently induce long-term, safe and specific antibodies against PCSK9 in BALB/C mice [38]. In this study, we explored the effects of this nanoliposomal antiPCSK9 vaccine in BALB/C mice bearing $4 \mathrm{~T} 1$ breast cancer. The present study is the first to evaluate in vivo efficacy and safety of PCSK9 inhibition in cancer cells.

\section{Material and methods}

\section{Vaccine preparation and characterization \\ Preparation and characterization of the liposome nanoparticles}

The lipid-film hydration method was used to prepare a nanoliposomal formulation containing 1,2-dimyristoyl-sn-glycero-3-phosphorylcholine (DMPC), 1,2-dimyristoyl-sn-glycero-3-phosphorylglycerol (DMPG) and cholesterol (Chol) (Avanti Polar Lipid; Alabaster, USA) at the final concentration of $40 \mathrm{mM}$ (total phospholipids and Chol). Briefly, DMPC, DMPG and Chol were dissolved in chloroform at the molar ratios of $75: 10: 15$, respectively. Lipid solution was dried to a thin lipid film under reduced pressure using rotary evaporation (Heidolph, Germany). The prepared lipid film was then freeze-dried (VD-800F, Taitech, Japan) overnight to completely remove the organic solvent. The dried lipids were then hydrated with 10 mM HEPES buffer ( $\mathrm{pH} 7.2)$ containing $5 \%$ dextrose, and vortexed and bath-sonicated to disperse completely into the buffer. To obtain small unilamellar vesicles (SUVs) with a uniform size of $100 \mathrm{~nm}$, the multilamellar vesicles (MLVs) were sequentially extruded using a mini-extruder (Avestin, Canada) with polycarbonate membranes of $600,400,200$ and $100 \mathrm{~nm}$ pore size, respectively. Particle size (diameter, $\mathrm{nm}$ ), zeta potential (surface charge, $\mathrm{mV}$ ) and polydispersity index (PDI) of the prepared nanoliposomal formulations were evaluated using dy- 
Table I. Sequence of immunogenic peptides used in the present study

\begin{tabular}{|lcl|}
\hline Peptide name & \multicolumn{1}{c|}{ Sequence } & Immunogenicity \\
\hline PCSK9 & S-I-P-W-N-L-E-R-I-T-P-V-R & B cell epitope \\
\hline Tetanus & A-Q-Y-I-K-A-N-S-K-F-I-G-I-T-E-L & T cell epitope \\
\hline IFPT & ${ }^{*}$ CGGGSIPWNLERITPVRAQYIKANSKFIGITEL & \\
\hline
\end{tabular}

*Bold amino acid codes are as a linker sequence for conjugating with DSPE-PEG-Mal. IFPT - immunogenic fused PCSK9-tetanus.

namic light scattering (DLS) technique on a Zetasizer (Nano-ZS, Malvern, UK) at room temperature (RT). The prepared nanoliposomes were stored at $4^{\circ} \mathrm{C}$ under argon.

\section{Immunogenic peptide}

The Immunogenic Fused PCSK9-Tetanus (IFPT) peptide was synthesized and purified by high performance liquid chromatography (HPLC) to a purity $>95 \%$ by ChinaPeptides Co., Ltd. (Shanghai, China). The previously designed IFPT construct [38] contains a PCSK9 peptide, as a B cell epitope inspired from the AFFiRiS group [35, 39], and a T-helper cell epitope belonging to the tetanus toxin used as a pharmaceutically acceptable carrier [40] (Table I). To attach the IFPT epitope on the surface of liposome nanoparticles, it was linked to DSPE-PEG-Mal (1,2-distearoyl-sn-glycero-3-phosphoethanolamine$N$-[maleimide(PEG)-2000]) lipid (Lipoid GmbH, Germany) via an $\mathrm{N}$-terminal cysteine residue added to IFPT peptide (Supplementary Figure S1).

\section{Construction of DSPE-PEG-IFPT micelles}

DSPE-PEG-maleimide lipid was used to link the immunogenic peptides on the surface of nanoliposomes as an adjuvant delivery system. The $\mathrm{N}$-terminal cysteine residue of the IFPT peptide provides a thiol group that reacts with the pyrrole group of maleimide and generates a thioether bond; thereby the peptide covalently binds to the DSPE-PEG-maleimide linker. The IFPT peptide and DSPE-PEG-Mal at the molar ratios of $1.2: 1$, respectively, were dissolved in DMSO/chloroform solution at the volume ratio of $1: 1$, and then gently stirred at RT for $48 \mathrm{~h}$. The linkage was confirmed using the TLC (thin layer chromatography) method with the mobile phase containing chloroform, methanol and water at the volume ratio of 82 : $16: 2$. Thereafter, the DMSO/chloroform solution was dried by a rotary evaporator and freeze-drying followed by hydration with sterile deionized water $(\mathrm{pH} 7.2)$ at $30^{\circ} \mathrm{C}$ to construct DSPE-PEG-IFPT micelles. The efficiency of the linkage in the prepared micelles was measured by HPLC analysis. The true value of the linked micelles was determined using efficiency of linkage and content of total lipid measured by the Bartlett phosphate assay method [41]

\section{HPLC analysis of the linkage efficiency}

The efficiency of linkage between the IFPT peptide and the DSPE-PEG-Mal linker was indirectly measured by HPLC quantification of the free peptide content of the prepared DSPE-PEGIFPT micelles. The HPLC apparatus was equipped with a Smart line HPLC Pump 1000, a PDA Detector 2800 (set at $220 \mathrm{~nm}$ ), and a Degasser5000, all from Knauer (Berlin, Germany). Each sample $(20 \mu \mathrm{l})$ was injected through a Smart line auto sampler and data were obtained and processed with ChromGate software (version 3.3.1) from Knauer (Berlin, Germany). Chromatographic separation was performed on a C18 reverse-phase column, $4.6 \mathrm{~mm} \times 25 \mathrm{~cm}$ (Shimadzu, Japan), using an isocratic mobile phase of $(0.1 \%$ TFA in water $) /(0.1 \%$ TFA in acetonitrile) at gradient ratios of $55 / 45$ to $45 / 55$ in $10 \mathrm{~min}$, at a flow rate of $1 \mathrm{ml} / \mathrm{min}$.

The IFPT peptide with HPLC purity > 95\% was used as a standard solution. The free peptide peak within the chromatogram of the micelle sample was identified and assigned based on the retention time $(2.2 \mathrm{~min})$ of the standard solution, followed by sample spiking.

To quantify the free peptide content of the micelle sample, a calibration curve was constructed by injecting standard solution at five concentrations $(50-500 \mu \mathrm{g} / \mathrm{ml})$; the curve was linear with a regression coefficient $\left(R^{2}\right)$ of 0.9954 . Using linear regression analysis of the calibration curve appearing in the standard chromatogram, the free peptide content of the micelle sample was estimated. Linkage efficiency in the constructed DSPE-PEG-IFPT micelles was calculated by subtracting the free peptide amount within the micelles quantified by HPLC from the amount of the IFPT peptide initially added.

\section{Construction and characterization of nanoliposomal IFPT vaccine}

Nanoliposomes were used as a delivery adjuvant to enhance immunogenicity of the peptide. Since many IFPT peptides can be conjugated to the surface of liposome nanoparticles, we propose that IFPT-linked nanoliposomes can elicit a high-titer antibody against self-antigen PCSK9, maybe through elevating peptide valency. To conjugate the IFPT peptide to the nanoliposome surface, the post-in- 
sertion approach was performed, in which the prepared DSPE-PEG-IFPT micelles $(100 \mu \mathrm{g}$, based on the linked peptide) and liposome nanoparticles $(1 \mathrm{ml})$ were mixed and then gently shaken at $45^{\circ} \mathrm{C}$ for $3 \mathrm{~h}$. The micelles are inserted in the nanoliposome bilayer via the DSPE phospholipid moiety, and expose IFPT peptides on the nanoliposome surface through the PEG chains. Particle size, surface charge and PDI of the prepared nanoliposomal IFPT particles were evaluated using DLS technique on a Zetasizer (Nano-ZS, Malvern, UK) at RT. The IFPT-conjugated nanoliposomes were adsorbed to $0.4 \%$ alum adjuvant (Sigma-Aldrich) at a $1: 1$ $(v: v)$ ratio in a total volume of $400 \mu \mathrm{l}$ and stored at $4^{\circ} \mathrm{C}$ under argon. Prior to injection, the nanoliposomal IFPT plus alum vaccine hereafter called L-IFPTA ${ }^{+}$ was brought to RT and carefully mixed.

\section{Animals and cell line}

A total of 20 female BALB/c mice (4-6 weeks old) were provided from Pasteur Institute of Tehran, Iran and fed with ad libitum access to purified water and a commercial stock diet. All mice were housed in a pathogen-free animal house at a temperature of $22 \pm 1^{\circ} \mathrm{C}$ with a $12: 12 \mathrm{~h}$ light : dark cycle and maintained under a relative humidity of $50 \pm 10 \%$. Animal care was performed in accordance with welfare guidelines established by the Institutional Ethical Committee and Research Advisory Committee of Mashhad University of Medical Sciences. At the end of the experiment all animals were euthanized by $\mathrm{CO}_{2}$ inhalation.

The 4T1 mammary carcinoma cell line was purchased from Pasteur Institute of Tehran, Iran and cultured in RPMI-1640 medium containing $10 \%$ FBS and supplemented with penicillin $(100 \mathrm{lU} / \mathrm{ml}) /$ streptomycin $(100 \mathrm{mg} / \mathrm{ml})$. The cells were incubated at $37^{\circ} \mathrm{C}$ with a $5 \% \quad \mathrm{CO}_{2} / 95 \%$ air humidified atmosphere.

\section{Vaccination program}

Following 1 week of taming prior to the experimental procedures, the mice were randomly classified into two groups: a vaccine group $(n=10)$ and an untreated group $(n=10)$ group. The immunization was primed at week 0 (W0) and followed by three boosters (W2, W4, and W6), in a bi-weekly interval in a subcutaneous manner (Figure 1), while untreated mice simultaneously received phosphate-buffered saline (PBS). Tail vein bleeding was performed 2 weeks after each vaccination for the titration of plasma anti-PCSK9 antibody.

\section{ELISA measurement of plasma anti-PCSK9 peptide antibody}

To determine the titer of anti-PCSK9 antibodies, plasma samples were collected and analyzed by the ELISA method. Briefly, PCSK9 peptide at the concentration of $5 \mu \mathrm{g} / \mathrm{ml}$ in $0.1 \mathrm{M} \mathrm{NaHCO}_{3}$ ( $\mathrm{pH}$ 9.2-9.4) was coated overnight in a 96-well Nunc-MaxiSorp plate. Free binding sites were then blocked by incubation with blocking buffer $(1 \times \mathrm{PBS}, 1 \% \mathrm{BSA})$ for $1 \mathrm{~h}$ at $37^{\circ} \mathrm{C}$. Diluted plasma ( 1 : 400 in dilution buffer $[1 \times \mathrm{PBS} / 0.1 \% \mathrm{BSA} / 0.05 \%$ Tween-20]) was added, serially diluted $1: 4$, and incubated for $1 \mathrm{~h}$ at $37^{\circ} \mathrm{C}$. Each ELISA plate contained a standard antibody as an internal control. For the detection, biotinylated anti-mouse IgG $(\mathrm{H}+\mathrm{L})$ (Sigma-Aldrich; $1: 1000)$ in $1 \times \mathrm{PBS} / 0.1 \%$ BSA $/ 0.1 \%$ Tween-20 was applied and incubated for $1 \mathrm{~h}$ at $37^{\circ} \mathrm{C}$. As a next step, horseradish peroxidase coupled to streptavidin (Roche) was added $\left(30 \mathrm{~min}, 37^{\circ} \mathrm{C}\right.$ ) followed by the addition of the substrate 2,2'-azinobis [3-ethylbenzothiazoline-6-sulfonic acid]-diammonium salt (ABTS) (Sigma-Aldrich) (15 min, RT). The optical density (OD) at $450 \mathrm{~nm}$ was measured with a microwell plate reader (Sunrise, Tecan, Switzerland) and the titers were defined as the dilution factor referring to $50 \%$ of the maximal optical density $\left(\mathrm{OD}_{\max } / 2\right)$. The mean titers \pm SD of all animals per group are presented.

\section{Plasma PCSK9 quantification}

Plasma PCSK9 concentration in the vaccinated mice was measured by CircuLex rat PCSK9 ELISA (Circulex, Cy-8078, MBL, Woburn, MA) according to the manufacturer's instructions. Briefly, $100 \mu \mathrm{l}$ of the diluted $1: 100$ plasma samples was added to a 96-well microplate and incubated for $1 \mathrm{~h}$ at RT. A HRP-conjugated anti-PCSK9 antibody was added for $1 \mathrm{~h}$ followed by the substrate reagent and stop solution, all at RT. Optical density was detected at $450 \mathrm{~nm}$ with a Microwell plate reader (Sunrise, Tecan, Switzerland). A standard curve provided by the supplier was defined to measure PCSK9 concentration.

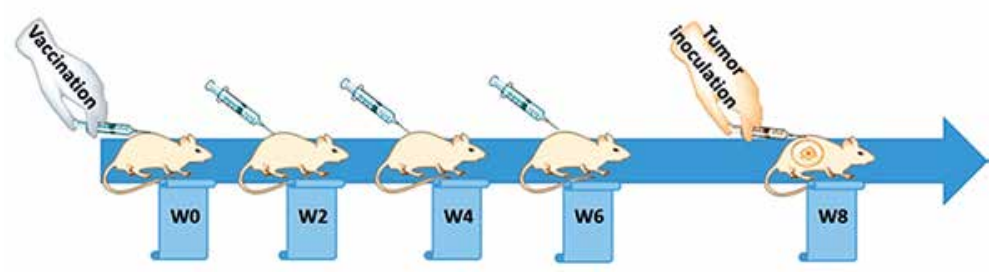

Figure 1. Study design 


\section{PCSK9 inhibition analysis}

To evaluate inhibition of mice PCSK9 by vaccine-induced antibodies, interaction of generated antibodies with PCSK9 was assayed. For this purpose, the same kit CircuLex PCSK9 ELISA was used, but instead of HRP-conjugated anti-PCSK9 antibody, detection was performed with HRP-conjugated anti-mouse IgG $(\mathrm{H}+\mathrm{L})$ (Sigma Aldrich; dilution 1 : 5000) incubated for $1 \mathrm{~h}$ at RT, followed by the substrate reagent and stop solution provided by the supplier. The OD was detected at $450 \mathrm{~nm}$ with the Microwell plate reader.

\section{In vitro evaluation of inhibiting PCSK9-LDLR interaction}

The CircuLex PCSK9-LDLR in vitro binding assay kit (CircuLex, Cy-8150, MBL, Woburn, MA) was employed to analyze the capacity of vaccine-generated antibodies for inhibition of the PCSK9LDLR interaction in vitro. Briefly, $100 \mu$ of the vehicle control or plasma samples of vaccinated mice was added to a 96-well microplate pre-coated with a recombinant LDLR-AB domain, which contains the binding site for PCSK9. Immediately after that, the reaction was initiated by adding a "Histagged PCSK9 wiled type" solution incubated for $2 \mathrm{~h}$ followed by adding a biotinylated anti-His-tag monoclonal antibody for $1 \mathrm{~h}$ at RT. Then, HRP-conjugated streptavidin was coated for $1 \mathrm{~h}$ at RT followed by the substrate reagent and stop solution. In this method, a higher amount of PCSK9-LDLR interaction is associated with higher ELISA OD, in which in the presence of anti-PCSK9 antibody this interaction is inhibited and consequently ELISA OD is decreased. A dose-response curve with appropriate serial dilutions of "His-tagged PCSK9 wild type" solution was drawn to measure the accurate inhibition percentage of test samples.

\section{Evaluation of in vivo anti-tumor efficacy}

Two weeks after the last booster, the vaccinated and unvaccinated BALB/c mice were subcutaneously inoculated with $4 \mathrm{~T} 1$ breast carcinoma cells $(5 \times$ $10^{5} / 50 \mu \mathrm{l} \mathrm{PBS}$ /mouse) into the right flank at day zero. Tumor growth was monitored at a 2-day interval by calculating the tumor volume after measuring three orthogonal diameters with calipers according to the formula: Tumor volume $\left(\mathrm{mm}^{3}\right)=($ length $\times$ height $\times$ width) $\times 0.52$. After the tumor mass was palpable (approximately $10 \mathrm{~mm}^{3}$ ) on day 10 , the mice were randomly divided into four groups (5 mice/group) and subjected to different treatment protocols: (1) the PBS (untreated control) group which involved unvaccinated tumor-bearing mice receiving a single tail vein injection of saline buffer, (2) the vaccine group which involved vaccinated tumor-bearing mice receiving a single tail vein injection of saline buffer, (3) the combination group which involved vaccinated tumor-bearing mice receiving a single tail vein injection of Doxil (15 mg/kg), and (4) the Doxil (positive control) group which involved unvaccinated tumor-bearing mice receiving a single tail vein injection of Doxil (15 mg/kg).

To evaluate therapeutic efficacy, mouse body weight, tumor size, general health, and survival were monitored every other day for 60 days. Animals' euthanasia ( $\mathrm{CO}_{2}$ inhalation) was performed in those with 4T1 tumor due to the following ethical considerations: body weight loss $>20 \%$ of initial mass, tumor volume greater than $2.0 \mathrm{~cm}$ in one dimension, or mice became sick and unable ambulate to reach food/water $[42,43]$. For each mouse, the time to reach tumor volume above $1000 \mathrm{~mm}^{3}$ or the time to reach the end point (TTE) as a response variable was calculated from the equation of the line obtained by exponential regression of the tumor growth curve. For each group, the percent of tumor growth delay (\%TGD) was determined by calculating the difference between the average TTE of the treatment group (T) and the average TTE of the control group (C), (\%TGD = $[(T-C) / C] \times 100)[44]$. For each treatment group, the percentage of increased life span (\%ILS) was measured based on the following formula: [(average survival time of treatment group/average survival time of control group $\times 100)-100]$ [45]

\section{Statistical analysis}

Statistical analysis was performed using SPSS Statistics version 20 and GraphPad Prism version 7.04 software. Survival data were analyzed using the log-rank (Mantel-Cox) test. Other comparisons were done using one-way ANOVA and Tukey post-hoc multiple comparison test. Values were expressed as mean \pm SD or median for normally and non-normally distributed data, respectively. Results with $p<0.05$ were considered as statistically significant.

\section{Results}

\section{Physical properties}

Characterization of liposome nanoparticles showed a size of $135 \mathrm{~nm}$ and $160 \mathrm{~nm}$ for free and IFPT-linked nanoliposomes, respectively. Other characteristics including PDI and zeta potential are detailed in Table II.

\section{Analysis of DSPE-PEG-IFPT micelles}

Attachment of the IFPT peptides to the DSPEPEG-Mal linker were confirmed qualitatively and quantitatively using TLC (Supplementary Figure S2) and HPLC (Supplementary Figure S3) methods, yielding an attachment yield of $96 \%$ according to HPLC. 
Table II. Physical properties of nanoliposomal formulations

\begin{tabular}{|lccc|}
\hline Formulation & $\begin{array}{c}\text { Z-average }[\mathrm{nm}] \\
\text { Mean } \pm \mathrm{SD}, \boldsymbol{n}=\mathbf{3}\end{array}$ & $\begin{array}{c}\text { Zeta potential [mV] } \\
\text { Mean } \pm \text { SD, } n=\mathbf{3}\end{array}$ & $\begin{array}{c}\text { PDI } \\
\text { Mean } \pm \text { SD, } n=\mathbf{3}\end{array}$ \\
\hline Empty nanoliposome & $135 \pm 9.5$ & $-40.9 \pm 4.8$ & $0.03 \pm 0.01$ \\
\hline IFPT linked-nanoliposome & $160 \pm 11.5$ & $-25.7 \pm 4.2$ & $0.136 \pm 0.08$ \\
\hline
\end{tabular}

PDI - polydispersity index.

PCSK9 antibody response in the vaccinated $\mathrm{BALB} / \mathrm{c}$ mice

The L-IFPTA ${ }^{+}$vaccine was found to induce high IgG titers against PCSK9 peptide in BALB/C mice upon 4 vaccinations in biweekly intervals. Further, long-term analysis revealed that anti-PCSK9 antibody titer reached peak levels at week 8 and remained constant up to week 10 , and then showed a decreasing trend (Figure $2 \mathrm{~A}$ ).

\section{Targeting of plasma PCSK9 by vaccine- induced antibodies}

Vaccine-induced PCSK9 antibodies targeted plasma PCSK9 in vaccinated mice in a specific manner. Plasma PCSK9 levels were $20 \pm 9 \mathrm{ng} / \mathrm{ml}$ and $63 \pm 13 \mathrm{ng} / \mathrm{ml}$ in the vaccinated and control groups, respectively (Figure $2 \mathrm{~B}$ ).

Plasma PCSK9 concentrations were decreased by $68 \%(-43 \pm 9 \mathrm{ng} / \mathrm{ml}, p=0.009)$ in the vaccinated mice as compared to control mice. The specificity of PCSK9 targeting by vaccine-induced antibodies was tested using an ELISA assay as described above and in our previous report [31].

A significantly higher $\mathrm{OD}_{450}$ signal was detected from the plasma of vaccinated mice, suggesting that vaccine-induced PCSK9 antibodies were able to directly bind to PCSK9 (Figure 2 C).
B

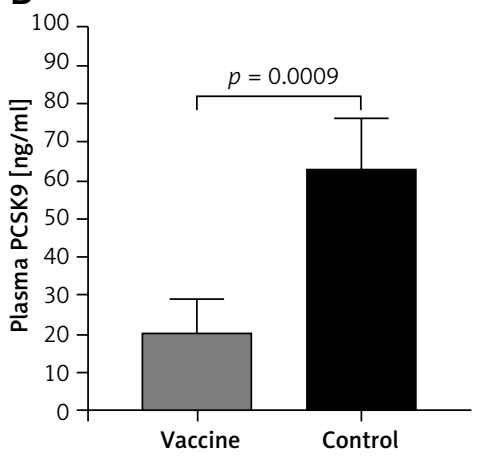

A

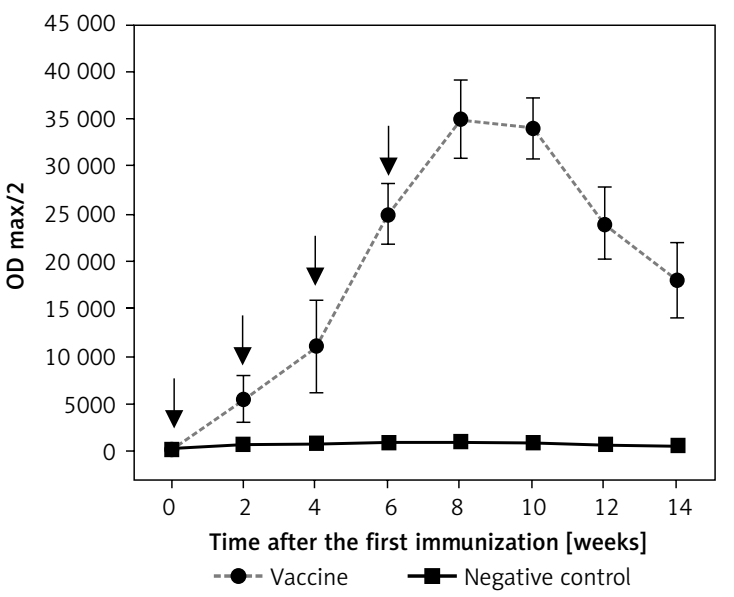

Figure 2. Anti-PCSK9 vaccine efficacy. A - Anti-PCSK9 antibody titers (ODmax/2) over 14 weeks after prime immunization, generated upon 4 immunizations at a bi-weekly interval (indicated by arrows). B - Plasma concentrations of PCSK9 in vaccine and control groups were $20 \pm 9 \mathrm{ng} / \mathrm{ml}$ and $63 \pm 13 \mathrm{ng} / \mathrm{ml}$, respectively. C - Direct detection of antibodies bound to plasma PCSK9 in blood samples from vaccinated and control mice. Increased OD450 is indicative for vaccine generated anti-PCSK9 antibodies which directly target PCSK9. D - In vitro PCSK9/LDLR binding assay. Plasma sample of vaccine group could decrease PCSK9 binding to LDLR by $46 \%$, compared with plasma sample of control group. Values are expressed as means \pm SD ( $n=3$ replicates of the pooled samples of 10 mice per group). Significance compared to control values was analyzed by unpaired 2-tailed Student's $t$-test 


\section{PCSK9-LDLR interaction blockade by vaccine-induced PCSK9 antibodies}

Using the above-mentioned in vitro assay, PCSK9 antibodies were found to inhibit the interaction between PCSK9 and LDLR. Binding of PCSK9 to LDLR was reduced by $46 \%$ in the presence of plasma obtained from L-IFPTA ${ }^{+}$-vaccinated mice, as compared to control mice (Figure $2 \mathrm{D}$ ).

\section{Efficacy of nanoliposomal antiPCSK9 in 4T1 breast cancer model treatment}

To evaluate the effect of PCSK9 inhibition on breast cancer progression, a 4T1 breast tumor model was induced in BALB/c mice formerly immunized with liposomal-antiPCSK9 vaccine. The prophylactic effect of the vaccine on tumor-bearing mice was assessed by monitoring body weight changes, tumor growth rate in terms of mean tumor size $\left(\mathrm{mm}^{3}\right)$, and survival. It was observed that two out of five vaccinated mice remained tumor-free. The weight monitoring curve showed that body mass was decreased in the vaccinated mice from 17 days after tumor induction. As compared with the control group, vaccinated mice showed significantly $(p<0.05)$ higher body weight loss from 32 days after tumor induction (Figure $3 \mathrm{~A}$ ). Monitoring the tumor size (Figure $3 \mathrm{~B}$ ) revealed that the tumor growth rate was partly but not significantly lower in the vaccinated mice than that in the control mice. Kaplan-Meier curves (Figure 3 C) revealed that the vaccinated mice survived slightly longer than control mice; however, this difference was not statistically significant ( $p<0.05$, log-rank test). To determine endpoint therapeutic modalities of antiPCSK9 vaccine in tumor-bearing $B A L B / c$ mice, time to reach endpoint (TTE) and percentage of tumor growth delay (TGD\%), as well as median survival time (MST) and increase in lifespan (ILS), were measured (Table III).

A

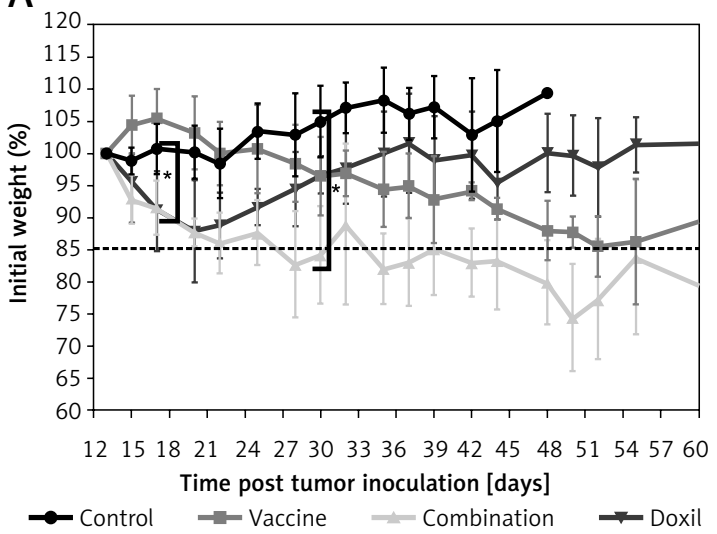

B

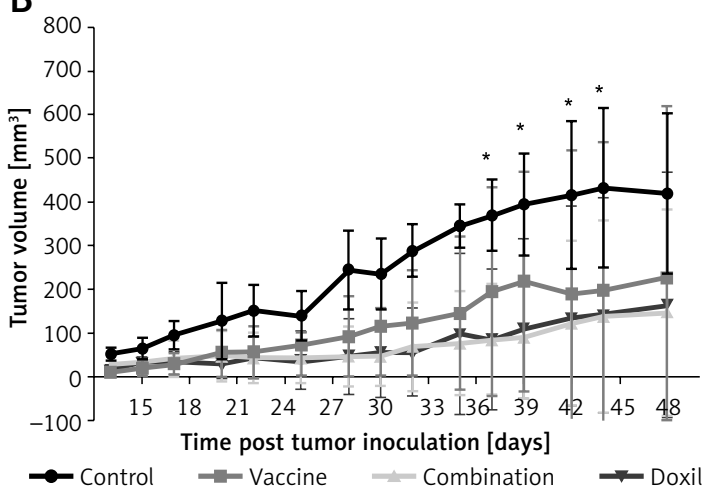

$\mathrm{C}$

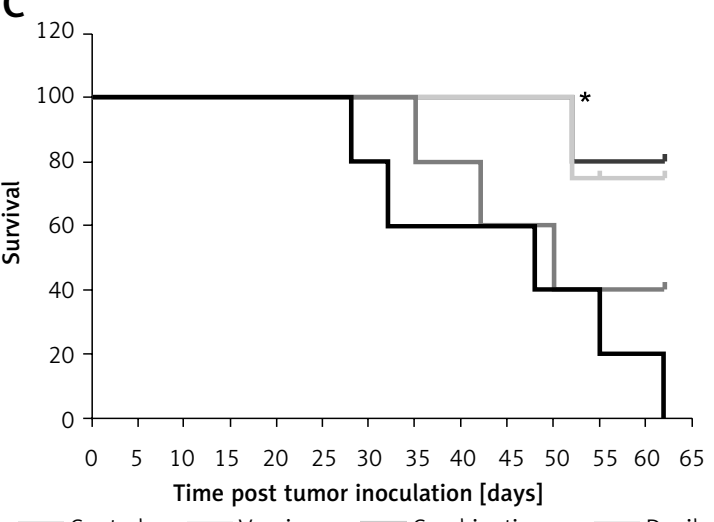

D

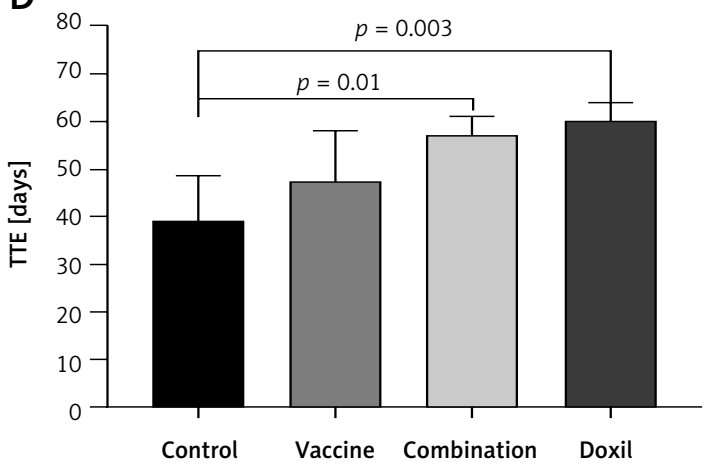

Figure 3. Therapeutic efficacy of vaccine and combination (vaccine plus Doxil) treatment was evaluated and compared with the control and Doxil (as the cytotoxic positive control) in a mouse model of 4T1 breast tumor. Values are presented as the mean $\pm \mathrm{SD}(n=5$ per group). A - Animal body weight was measured every 2 days. *A significant difference $(p<0.05)$ of body weight loss between control group with Doxil and combination from day 17 , as well as between all groups from day 30 up to day 60. B - Tumor volume $\left(\mathrm{mm}^{3}\right)$ was measured every 2 days. ${ }^{*}$ A significant difference $(p<0.05)$ of average tumor volume between control group with Doxil and combination at days 37, 39, 42, 44. C - Kaplan-Meier curves show the survival rate of treatment and control group. *A significant difference $(p<0.05)$ between control group with Doxil and combination group. $\mathbf{D}-$ Measuring the time to reach endpoint (TTE) or the time to reach tumor volume above $1000 \mathrm{~mm}^{3}$ showed a significant difference between control group with combination and Doxil group 
Table III. Therapeutic efficacy data of different treatments in BALB/c mice bearing 4T1 tumor

\begin{tabular}{|lcccc|}
\hline Groups & TTE, mean \pm SD [days] & TGD (\%) & MST [days] & ILS (\%) \\
\hline Control & $39 \pm 9$ & - & 48 & - \\
\hline Vaccine & $47 \pm 10$ & 21.2 & 50 & 4.2 \\
\hline Doxil & $60 \pm 4$ & 53.8 & ND & ND \\
\hline Combination & $57 \pm 4$ & 48.1 & ND $^{*}$ & ND \\
\hline
\end{tabular}

TTE - time to reach end point, TGD - tumor growth delay, MST - median survival time, ILS - increase of life span.

${ }^{*}$ Not defined; survival did not reach below $50 \%$ at the determined time point, then median survival could not be computed

The TTE of the vaccine and control groups were $47 \pm 10$ and $39 \pm 9$ days, respectively. TGD as a widely accepted method for assessment of tumor treatment efficacy showed that the tumor growth rate in the vaccine group was reduced by $21.2 \%$ compared with the control group. The MSTs of the vaccine and control groups were 50 and 48 days, respectively. The ILS revealed that the vaccinated mice's life was prolonged by $4.2 \%$ compared with the control mice.

\section{Synergistic antitumor effect of} nanoliposomal antiPCSK9 vaccine and Doxil

To evaluate the effect of PCSK9 inhibition on tumor chemotherapy, the vaccinated group received a single dose of Doxil (called the combination group) and was compared with the groups of mice that received vaccine and Doxil alone. Two out of five mice in the combined treatment group remained tumor-free. As compared with the Doxil group, the combined treatment mice showed significantly $(p<0.05)$ higher body weight loss from 28 days after tumor induction. The body weight loss was found to be significantly higher in the combined treatment group than the vaccine group from 15 days after tumor induction. Additionally, 35 days after tumor inoculation, mice in the combined treatment group showed a body weight loss below the criterion for euthanasia - more than $20 \%$ weight loss as compared to the initial body weight at the day of tumor inoculation (Figure $3 \mathrm{~A}$ ). As demonstrated by tumor size analysis, tumor growth rate in the combination and Doxil groups was found to have an approximately similar trend; however, both groups showed a slightly but not significantly lower tumor growth rate during the experiment compared with the vaccine group (Figure $3 \mathrm{~B}$ ). The TTEs of the combination and Doxil groups were $57 \pm 4$ and $60 \pm 4$ days, respectively. The TGD\% showed that the tumor growth rate in the combination and Doxil groups was reduced by $48.1 \%$ and $53.8 \%$, respectively, as compared with the control group (Table III). Kaplan-Meier curves analysis using log-rank test (Figure $3 \mathrm{C}$ ) showed that the combination and Doxil groups survived significantly longer $(p<0.001)$ than the vaccine and control groups (Table III).

\section{Discussion}

Our recent study has shown that the nanoliposomal antiPCSK9 vaccine can directly target and inhibit circulating PCSK9 and efficiently decrease elevated levels of plasma LDL-C in mice [38]. Inconsistent associations between circulating lipids and risk of breast cancer have been reported by observational and Mendelian randomization studies [9-16]. Nonetheless, there is no evidence on the effects of PCSK9 inhibitors on breast cancer occurrence. To explore whether PCSK9 inhibition is harmless or harmful in breast cancer, in the present in vivo study, effects of PCSK9 inhibition on breast cancer were evaluated for the first time. We found that the nanoliposomal antiPCSK9 $\left(\right.$ L-IFPTA $\left.^{+}\right)$vaccine, as a PCSK9 inhibitor, could moderately, but not significantly, decrease tumor growth in the mice bearing 4T1 breast cancer. Our present results revealed that the L-IFPTA ${ }^{+}$vaccine could provoke functional anti-PCSK9 antibodies (Figure $2 \mathrm{~A}$ ) targeting circulating PCSK9 (Figure 2 C), thereby reducing its plasma level (Figure 2 B) and activity (Figure 2 D) in BALB/C mice. The induced antiPCSK9 antibodies were found to reduce the rate of tumor growth by $21.2 \%$, and to prolong lifespan by $4.2 \%$ in vaccinated mice bearing breast cancer (Figure 3 and Table III). Although the results were not statistically significant, these findings suggest that PCSK9 inhibition at least exerts no side effect on breast tumor endpoint.

The slight anti-tumor effect of PCSK9 inhibition observed in our study can be supported by a recent Mendelian randomization study which showed that LDL-lowering variants mimicking PCSK9 inhibitors had a significant association with lower risk of breast cancer occurrence, whereas PCSK9 LDL-raising genetic variants of the PCSK9 gene were associated with a higher risk of breast cancer [16]. Likewise, data from a prospective population-based cohort study demonstrated that LDL-lowering PCSK9 variants cannot increase the risk of cancer [46]. Accordingly, a phenome-wide association analysis of LDL-lowering genetic variants in the PCSK9 locus mimicking PCSK9 inhibitors demonstrated no association between PCSK9 and risk of cancer in the UK Biobank sample [47].

As a second aim, we evaluated whether PCSK9 can affect cancer chemotherapy modalities. It was 
found that combined treatment of L-IFPTA ${ }^{+}$vaccine and Doxil could significantly decrease the rate of tumor growth by $48.1 \%$ compared with the untreated control. Likewise, the lifespan of mice was significantly increased in the combination and Doxil groups compared with the vaccine and control group.

In conclusion, our findings revealed that PCSK9 inhibition not only exerts no harmful effects but also could somewhat improve breast cancer behavior in an experimental model. This can suggest safety and efficacy of PCSK9 inhibitors in conditions other than cardiovascular disease such as cancer. However, further preclinical and clinical trials are necessary to verify the efficacy in terms of cancer incidence and progression and safety of therapeutic inhibition of PCSK9.

\section{Acknowledgments}

We are thankful for the financial support from the Mashhad University of Medical Sciences, Mashhad, Iran (grant number: 941726) and the National Institute for Medical Research Development (NIMAD), Tehran, Iran (Grant no: 963401).

We would also like to thank Mrs. Azam Abbasi and Mrs. Zahra Saberi for their valuable technical assistance.

\section{Conflict of interest}

The authors declare no conflict of interest.

\section{References}

1. Mehta LS, Watson KE, Barac A, et al. Cardiovascular disease and breast cancer: where these entities intersect: a scientific statement from the American Heart Association. Circulation 2018; 137: e30-66.

2. Antalis CJ, Uchida A, Buhman KK, Siddiqui RA. Migration of MDA-MB-231 breast cancer cells depends on the availability of exogenous lipids and cholesterol esterification. Clin Expl Metastasis 2011; 28: 733-41.

3. Nomura DK, Long JZ, Niessen S, et al. Monoacylglycerol lipase regulates a fatty acid network that promotes cancer pathogenesis. Cell 2010; 140: 49-61.

4. Menendez JA, Lupu R. Fatty acid synthase and the lipogenic phenotype in cancer pathogenesis. Nature Rev Cancer 2007; 7: 763-77.

5. Clayman RV, Gonzalez R, Elliott AY, et al. Cholesterol accumulation in heterotransplanted renal cell cancer. J Urol 1983; 129: 621-4.

6. Swyer G. The cholesterol content of normal and enlarged prostates. Cancer Res 1942; 2: 372-5.

7. dos Santos CR, Domingues G, Matias I, et al. LDL-cholesterol signaling induces breast cancer proliferation and invasion. Lipids Health Dis 2014; 13: 16.

8. Antalis CJ, Arnold T, Rasool T, et al. High ACAT1 expression in estrogen receptor negative basal-like breast cancer cells is associated with LDL-induced proliferation. Breast Cancer Res Treat 2010; 122: 661-70.

9. Touvier M, Fassier P, His M, et al. Cholesterol and breast cancer risk: a systematic review and meta-analysis of prospective studies. Br J Nutr 2015; 114: 347-57.
10. Ni H, Liu H, Gao R. Serum lipids and breast cancer risk: a meta-analysis of prospective cohort studies. PLoS One 2015; 10: e0142669.

11. Chandler PD, Song Y, Lin J, et al. Lipid biomarkers and long-term risk of cancer in the Women's Health Study-3. Am J Clin Nutr 2016; 103: 1397-407.

12. His M, Zelek L, Deschasaux M, et al. Prospective associations between serum biomarkers of lipid metabolism and overall, breast and prostate cancer risk. Eur J Epidemiol 2014; 29: 119-32.

13. Benn M, Tybjærg-Hansen A, Stender S, et al. Low-density lipoprotein cholesterol and the risk of cancer: a mendelian randomization study. J Natl Cancer Inst 2011; 103: 508-19.

14. Islam MM, Yang HC, Nguyen PA, et al. Exploring association between statin use and breast cancer risk: an updated meta-analysis. Arch Gynecol Obstet 2017; 296: 1043-53.

15. Trialists CT. Lack of effect of lowering LDL cholesterol on cancer: meta-analysis of individual data from 175,000 people in 27 randomised trials of statin therapy. PLoS One 2012; 7: e29849.

16. Nowak C, Ärnlöv J. A Mendelian randomization study of the effects of blood lipids on breast cancer risk. Nat Commun 2018; 9: 3957.

17. Lambert G, Sjouke B, Choque B, et al. The PCSK9 decade thematic review series: new lipid and lipoprotein targets for the treatment of cardiometabolic diseases. J Lipid Res 2012; 53: 2515-24.

18. Qian YW, Schmidt RJ, Zhang Y, et al. Secreted PCSK9 downregulates low density lipoprotein receptor through receptor-mediated endocytosis. J Lipid Res 2007; 48: 1488-98.

19. Abifadel M, Varret M, Rabès JP, et al. Mutations in PCSK9 cause autosomal dominant hypercholesterolemia. Nat Genet 2003; 34: 154-6.

20. Cohen J, Pertsemlidis A, Kotowski IK, et al. Low LDL cholesterol in individuals of African descent resulting from frequent nonsense mutations in PCSK9. Nat Genet 2005; 37: 161-5.

21. Zhao Z, Tuakli-Wosornu Y, Lagace TA, et al. Molecular characterization of loss-of-function mutations in PCSK9 and identification of a compound heterozygote. Am J Hum Genet 2006; 79: 514-23.

22. Hooper AJ, Marais AD, Tanyanyiwa DM, Burnett JR. The C679X mutation in PCSK9 is present and lowers blood cholesterol in a Southern African population. Atherosclerosis 2007; 193: 445-8.

23. Sabatine MS, Giugliano RP, Keech AC, et al. Evolocumab and clinical outcomes in patients with cardiovascular disease. N Engl J Med 2017; 376: 1713-22.

24. Schwartz GG, Steg PG, Szarek M, et al. Alirocumab and cardiovascular outcomes after acute coronary syndrome. N Engl J Med 2018; 379: 2097-107.

25. Sahebkar A, Watts GF. New therapies targeting apoB metabolism for high-risk patients with inherited dyslipidaemias: what can the clinician expect? Cardiovasc Drugs Ther 2013; 27: 559-67.

26. Sahebkar A, Watts GF. New LDL-cholesterol lowering therapies: pharmacology, clinical trials, and relevance to acute coronary syndromes. Clin Ther 2013; 35: 1082-98.

27. Banach M, Penson PE. What have we learned about lipids and cardiovascular risk from PCSK9 inhibitor outcome trials: ODYSSEY and FOURIER? Cardiovasc Res 2019; 115: e26-31.

28. Dragan S, Serban MC, Banach M. Proprotein convertase subtilisin/kexin 9 inhibitors: an emerging lipid-lowering therapy? J Cardiovasc Pharmacol Ther 2015; 20: 157-68. 
29. Soran H, Adam S, Mohammad JB, et al. Hypercholesterolaemia - practical information for non-specialists. Arch Med Sci 2018; 14: 1-21.

30. Jaworski K, Jankowski P, Kosior DA. PCSK9 inhibitors from discovery of a single mutation to a groundbreaking therapy of lipid disorders in one decade. Arch Med Sci 2017; 13: 914-29.

31. Food and Drug Administration. FDA approves praluent to treat certain patients with high cholesterol. In. 2015.

32. Robinson JG, Farnier M, Krempf $M$, et al. Efficacy and safety of alirocumab in reducing lipids and cardiovascular events. N Engl J Med 2015; 372: 1489-99.

33. Food and Drug Administration. FDA approves Repatha to treat certain patients with high cholesterol. In. 2015.

34. Sabatine MS, Giugliano RP, Wiviott SD, et al. Efficacy and safety of evolocumab in reducing lipids and cardiovascular events. N Engl J Med 2015; 372: 1500-9.

35. Galabova G, Brunner S, Winsauer G, et al. Peptide-based anti-PCSK9 vaccines-an approach for long-term LDLC management. PLoS One 2014; 9: e114469.

36. Fattori E, Cappelletti M, Surdo PL, et al. Immunization against proprotein convertase subtilisin-like/kexin type 9 lowers plasma LDL-cholesterol levels in mice. J Lipid Res 2012; 53: 1654-61.

37. Crossey E, Amar MJ, Sampson M, et al. A cholesterol-lowering VLP vaccine that targets PCSK9. Vaccine 2015; 33: 5747-55.

38. Momtazi-Borojeni AA, Jaafari MR, Badiee A, Sahebkar A. Long-term generation of antiPCSK9 antibody using a nanoliposome-based vaccine delivery system. Atherosclerosis 2019; 283: 69-78.

39. Schneeberger A, Mandler M, Otava O, et al. Development of AFFITOPE vaccines for Alzheimer's disease (AD) - from concept to clinical testing. J Nutr Health Aging 2009; 13: 264-7.

40. Slingluff CL, Yamshchikov G, Neese P, et al. Phase I trial of a melanoma vaccine with gp100280-288 peptide and tetanus helper peptide in adjuvant: immunologic and clinical outcomes. Clin Cancer Res 2001; 7: 3012-24.

41. Bartlett GR. Phosphorus assay in column chromatography. J Biol Chem 1959; 234: 466-8.

42. Huang Z, Szoka Jr FC. Sterol-modified phospholipids: cholesterol and phospholipid chimeras with improved biomembrane properties. J Am Chem Soc 2008; 130: 15702-12.

43. Huang Z, Jaafari MR, Szoka Jr FC. Disterolphospholipids: nonexchangeable lipids and their application to liposomal drug delivery. Angewandte Chemie 2009; 121: 4210-3.

44. Schluep T, Hwang J, Cheng J, et al. Preclinical efficacy of the camptothecin-polymer conjugate IT-101 in multiple cancer models. Clin Cancer Res 2006; 12: 1606-14.

45. Huang SK, Mayhew E, Gilani S, et al. Pharmacokinetics and therapeutics of sterically stabilized liposomes in mice bearing C-26 colon carcinoma. Cancer Res 1992; 52: 6774-81.

46. Folsom AR, Peacock JM, Boerwinkle E. Sequence variation in proprotein convertase subtilisin/kexin type 9 serine protease gene, low LDL cholesterol, and cancer incidence. Cancer Epidemiol Prev Biomarkers 2007; 16: 2455-8.

47. Schmidt AF, Holmes MV, Preiss D, et al. Phenome-wide association analysis of LDL-cholesterol lowering genetic variants in PCSK9. bioRxiv 2018; 329052. 